Unfortunately, the information regarding current neuroleptic medication was not helpful as the authors failed to supply the formula for conversion to chlorpromazine equivalents. Of the formulae in common use, there is reasonable agreement on the relative potencies of the oral neuroleptics, except for haloperidol. There are, however, large discrepancies between their recommendations for depot neuroleptics (Foster, 1989). The advantage of expressing the current medication in mean chlorpromazine equivalents is that some comparison can be made between clinical practices. The disadvantage is that, without additional information, such a cross-sectional measure has limited value in identifying patients at risk of developing obesity (Silverstone et al, Journal, August 1988, 153, 214-217), which is a medium to long-term complication.

FOSTER, P. (1989) Neuroleptic equivalence. Pharmaceutical Journal, 30 September, $431-432$.

The Stonebridge Research Centre

JUSTIN HAY

Cardiff Street

Carlton Road

Nottingham NG3 2FH

\section{Parental bonding}

SIR: Dr Birtchnell's inference (Journal, March 1993, 162, 335-344) that a poor early relationship with parents may extend into adult life cannot be supported by the data presented. To use a single measure from an already reductionistic and simple questionnaire (Parker, 1990) does not allow a generalisation to the total parental relationship. Greater support for Bowlby's hypothesis may have been offered if both dimensions of the Parental Bonding Instrument, that is care and overprotection, were used and if subjects were assigned quadrants of bonding (Parker, 1989). A comparable three-dimensional model of current relationships has already been well described in this population (Birtchnell, Journal, May 1991, 158, 648-657).

Parker, G. (1989) The parental bonding instrument: psychometric properties reviewed. Psychiatric Developments, 4, 317-335. - (1990) The parental bonding instrument. A decade of research. Social Psychiatry and Psychiatric Epidemiology, 25, 281-282.

\author{
Ashworth Hospital North \\ Park Lane \\ Maghull \\ Liverpool L31 $1 \mathrm{HW}$
}

The influence of ethnicity and family structure on relapse in first-episode schizophrenia

SIR: We read with great interest the article by Birchwood et al (Journal, December 1992, 161, 783-790) on family support systems within different ethnic populations. In a retrospective study, that we are currently undertaking, on patients of Asian background (i.e. immigrants from south-east Asia) we found a lower recidivism rate of readmission in the Asian patients, compared with a matched Caucasian sample. This is similar to Birchwood et al s findings.

An interesting finding in our study was that $47 \%$ of the Asian sample were on depot antipsychotics. A lower relapse rate was noted among Asian immigrants who were on depot antipsychotics, who had follow up from doctors and mental health workers of the same or similar ethnic backgrounds, and who had a strong family support system. These factors also contributed to better patient compliance with oral medications.

We concur with the conclusion by Birchwood et al with regard to language factors and diagnostic caution. Of the 30 patients in our sample, 15 had a poor command, or no knowledge of English. The frequency of a diagnosis of schizophrenia in these 15 patients was over $60 \%$. We feel that language factors in assessing these patients could have affected diagnostic validity. We agree that prospective studies and comparisons with samples from the country of origin would contribute to a better understanding of mental illness within an immigrant population.

Hari N. GarbHarRan TERRY ZIBIN

Alberta Hospital Ponoka

Box 1000

Ponoka, Alberta T4J IR6

\section{Tattooed female psychiatric patients}

AUTHORS' REPLY: We read with interest the response to our letter (Inch \& Huws, Journal, January 1993, $162,128-129)$. Sexual abuse in childhood has been shown to be positively correlated with symptoms and diagnosis of borderline personality disorder (Sheldon, Journal, January 1988, 152, 107-111), and there is a recognised association between tattoos and borderline personality disorder (Virkunen, 1976). This could suggest a correlation between childhood sexual abuse and tattoos.

We agree with the comment that on the basis of our four cases it is not possible at present to substantiate this hypothesis, and further work with a larger number of tattooed females with different psychiatric diagnoses should be undertaken. We feel that if 